\title{
The Molecule Cloud - compact visualization of large collections of molecules
}

\author{
Peter Ertl ${ }^{*}$ and Bernhard Rohde
}

\begin{abstract}
Background: Analysis and visualization of large collections of molecules is one of the most frequent challenges cheminformatics experts in pharmaceutical industry are facing. Various sophisticated methods are available to perform this task, including clustering, dimensionality reduction or scaffold frequency analysis. In any case, however, viewing and analyzing large tables with molecular structures is necessary. We present a new visualization technique, providing basic information about the composition of molecular data sets at a single glance.

Summary: A method is presented here allowing visual representation of the most common structural features of chemical databases in a form of a cloud diagram. The frequency of molecules containing particular substructure is indicated by the size of respective structural image. The method is useful to quickly perceive the most prominent structural features present in the data set. This approach was inspired by popular word cloud diagrams that are used to visualize textual information in a compact form. Therefore we call this approach "Molecule Cloud". The method also supports visualization of additional information, for example biological activity of molecules containing this scaffold or the protein target class typical for particular scaffolds, by color coding. Detailed description of the algorithm is provided, allowing easy implementation of the method by any cheminformatics toolkit. The layout algorithm is available as open source Java code.

Conclusions: Visualization of large molecular data sets using the Molecule Cloud approach allows scientists to get information about the composition of molecular databases and their most frequent structural features easily. The method may be used in the areas where analysis of large molecular collections is needed, for example processing of high throughput screening results, virtual screening or compound purchasing. Several example visualizations of large data sets, including PubChem, ChEMBL and ZINC databases using the Molecule Cloud diagrams are provided.
\end{abstract}

Keywords: Molecule cloud, Visualization, Scaffold analysis, Chemical databases, Open source

\section{Background}

One of the most typical tasks that a cheminformatics expert in pharmaceutical or agrochemical industry performs practically daily is analyzing and visualizing large collections of molecules. Typical areas, where this is needed are the analysis of the company compound archive and its enhancement by purchasing additional molecules from commercial compound providers, analysis of high-throughput screening results, design of combinatorial libraries, chemogenomics analysis of bioactivity data and many others [1]. But also researchers in academia are facing similar challenges when they need to

\footnotetext{
* Correspondence: peter.ertl@novartis.com

Novartis Institutes for BioMedical Research, Novartis Campus, CH-4056, Basel,
} Switzerland

process and visualize large molecular databases that have become freely available in the last few years [2] or were even generated in silico [3]. A number of methods to analyze and visualize large collections of molecules exist [4]. Among the most commonly used ones are various clustering techniques, dimensionality reduction approaches [5] or methods based on substructure analysis, particularly the analysis of molecule scaffolds [6]. But even if such sophisticated methods are applied, at the end it is necessary to visually inspect large tables of molecular structure diagrams.

The necessity to analyze and visualize large data sets, is of course, nothing specific to chemistry. Practically all areas of science need to cope with this problem. A simple, but very efficient method to analyze large data sets has been introduced in text processing. A method called 
"text cloud" or "word cloud" identifies the most common words in a text and displays them in form of a cloud diagram, where the frequency of a word is indicated by the size of the font used to render it. The most common words in the text like "and", "or" or "the" do not contain useful information and are therefore not displayed. This type of diagram allows very efficient and compact visualization of the most important "message" contained in the text (Figure 1). The word cloud graphs summarizing the content of web pages become one of

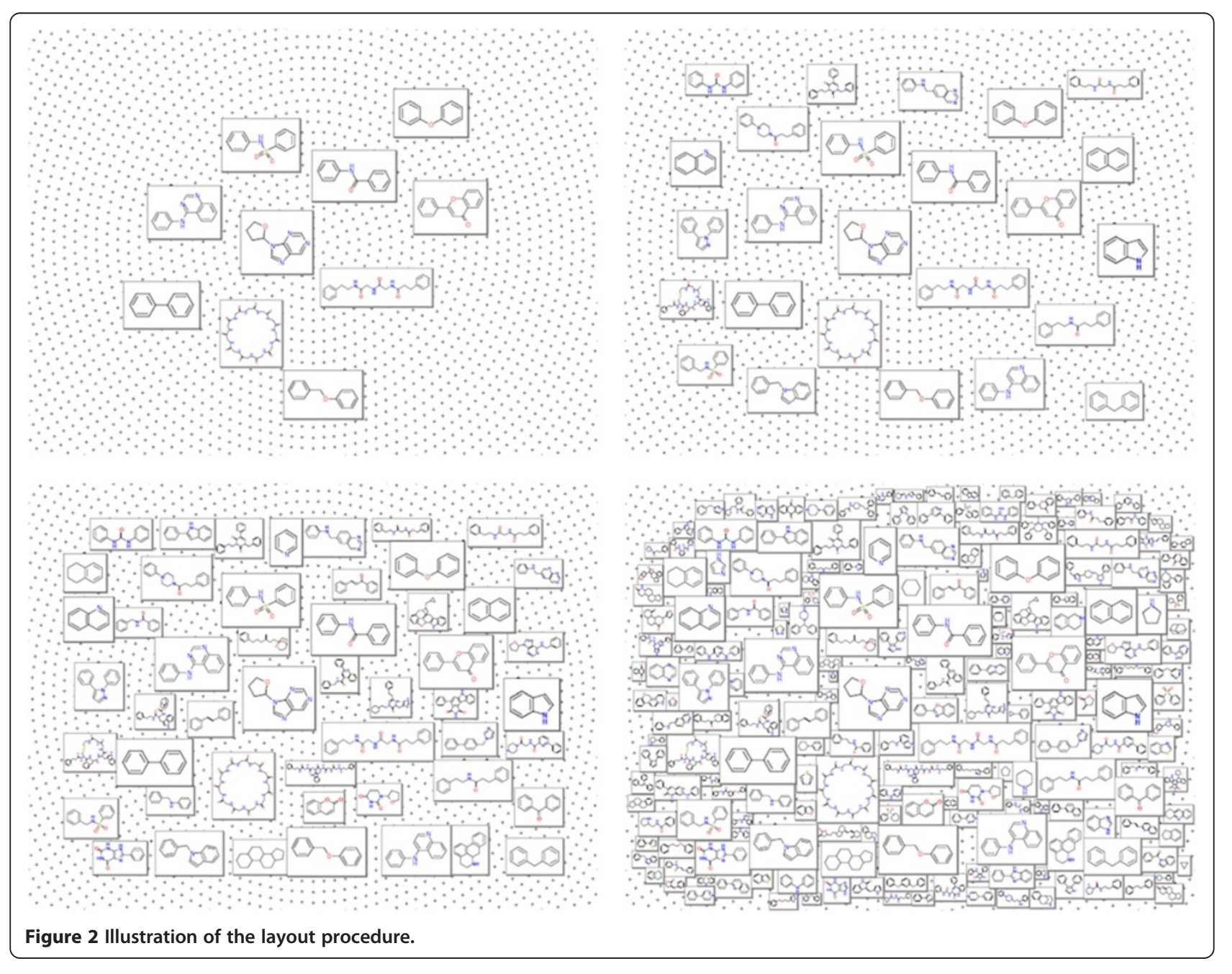




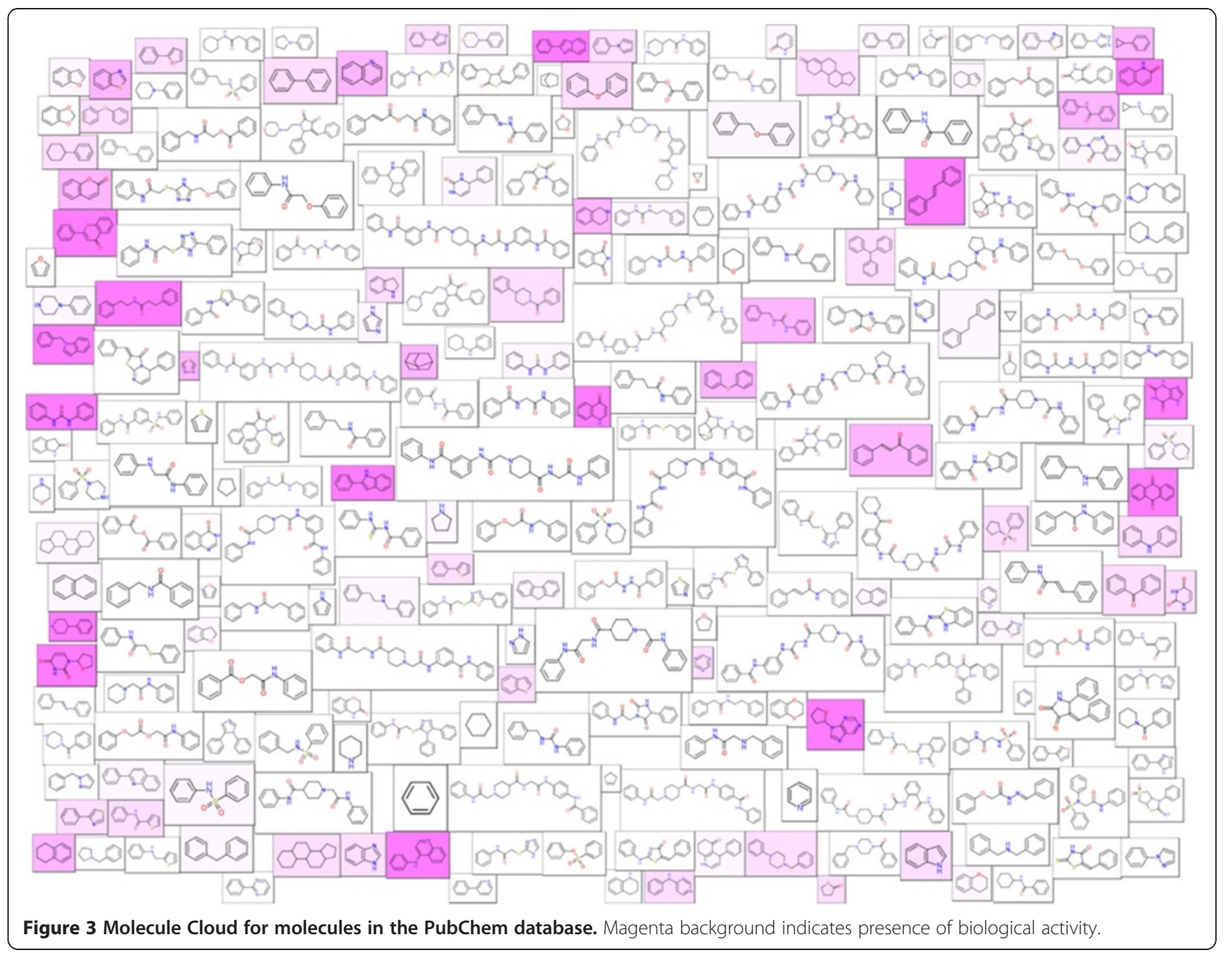

the symbols of Web 2.0. Several types of word cloud diagrams exist, differing in their layout and the way how the text is rendered.

The word cloud diagrams inspired us to develop a method for compact visualization of large molecular data sets. The method is based on the same principle, but instead of words molecular structures are displayed in the graph. The size of molecules corresponds to their frequency in the particular data sets. The largest structures catch the eyes of the observer first and therefore a single look at the diagram provides basic, condensed information about the content of the parent data set. In analogy with the "word cloud" we call this method "Molecule Cloud".

\section{Results and discussion}

\section{Molecule Cloud methodology}

The principle of the Molecule Cloud method is very simple. The most common substructure features present in the analyzed data set are identified and then displayed in such a way that their size corresponds to their frequency. The most common substructures are rendered the largest and therefore immediately catch the eye of an observer. Molecules are represented by their scaffolds, i.e. cores remaining when all non-ring substituents are removed. The concept of scaffold as the central part of a molecule is one of the basic concepts of medicinal chemistry and scaffolds play an important role in several drug discovery techniques like combinatorial chemistry and scaffold hopping [7]. Molecules without any rings are represented by their major chain, i.e. the longest chain, containing the largest number of heteroatoms. Reduction of molecules to scaffolds simplifies the analysis considerably. For example 35 million molecules from the PubChem database are represented by about 3.9 million scaffolds (50\% of which are singletons, present only once in the database) and about 150 thousand chains.

Once the most common scaffolds and chains are identified, they need to be visualized with their size scaled according to the frequency in the parent database. It is well known that the frequency of various substructure 


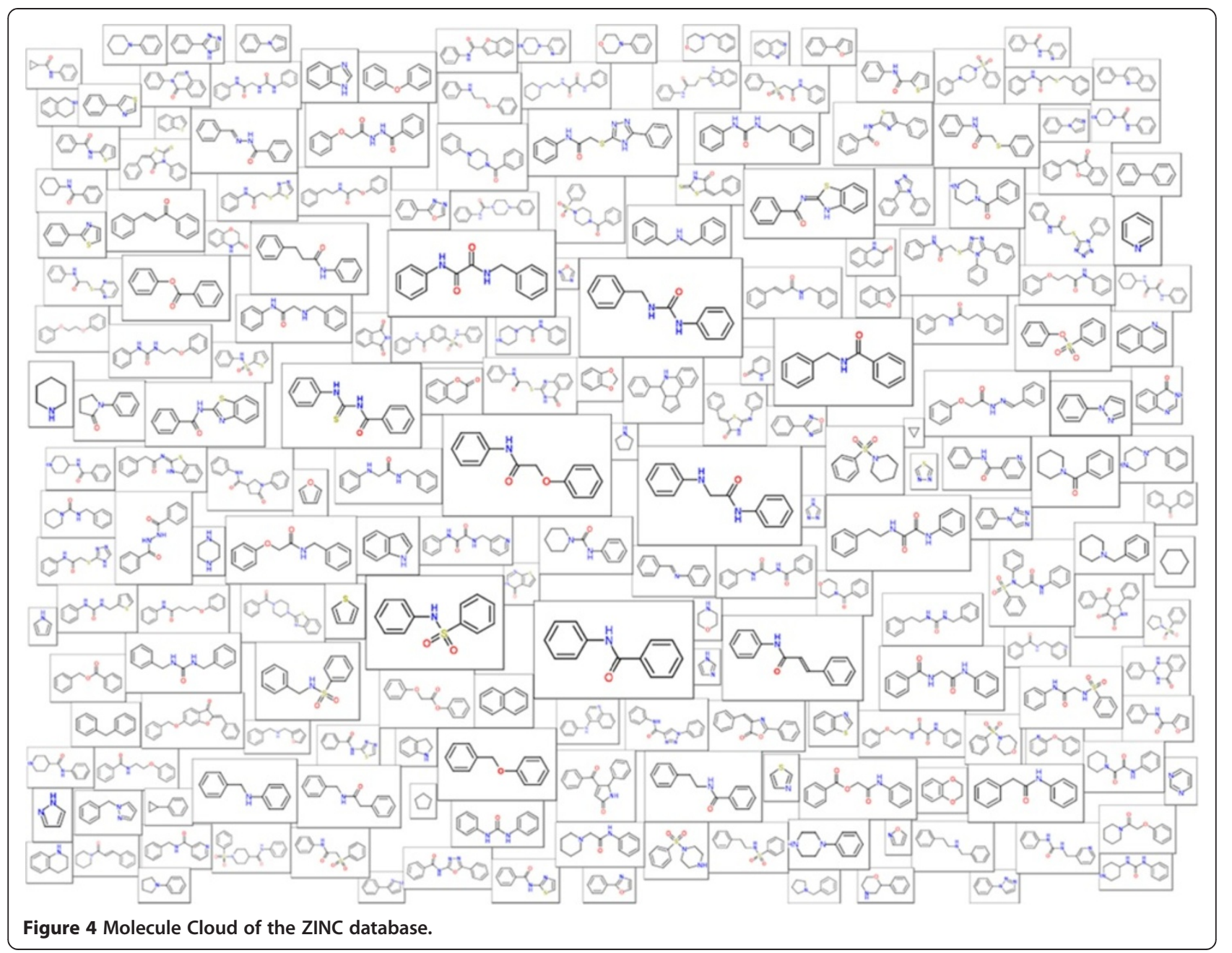

features like scaffolds, substituents or linkers in molecular databases follows the power law (so called "long tail" distribution) [8]. This means that only few scaffolds in a database are very common, while there are many rare scaffolds including large number of singletons. Before using scaffold frequencies as a scaling factor, they therefore need to be transformed into the logarithmic scale. Benzene is a special case, practically in all large data sets the benzene is clearly the most frequent scaffold. In many cases it is therefore advisable not to display it. Even after logarithmic transformation of frequencies benzene would be disproportionally large, and it would not contribute any useful information. Removal of benzene is similar to the removal of the common stop words in classical text clouds.

According to our experience the optimal number of substructures to be displayed in the Molecule Cloud is between 100 and 250. This number usually contains 30 50 large structures, easily recognizable, the rest are smaller structures that optically fill the image. Of course, when displaying the graph in a larger area (for example as a poster) the number of structures that can be displayed is proportionally larger.

The greatest challenge in generating the Molecule Cloud is the esthetically pleasing layout of molecules in the graph. This is done by a two-pass layout algorithm developed specially for this purpose. In the first step molecules are placed in the display area in such a way that their overlap is minimal. The process starts by sorting molecule images according to their size and placing the largest one in the center. Then other molecules, one by one are placed in a loop on a dense grid of predefined layout points and an "overlap score" is calculated for each placement. At the end the molecule is placed at the position with the best score (i.e. position where the overlap with other structures is minimal) and the next molecule is processed. This procedure is illustrated in the Figure 2, where one can see the layout after placement of 10, 25, 50 and 200 molecules. The "overlap score" used to identify the best position to place a molecule is 


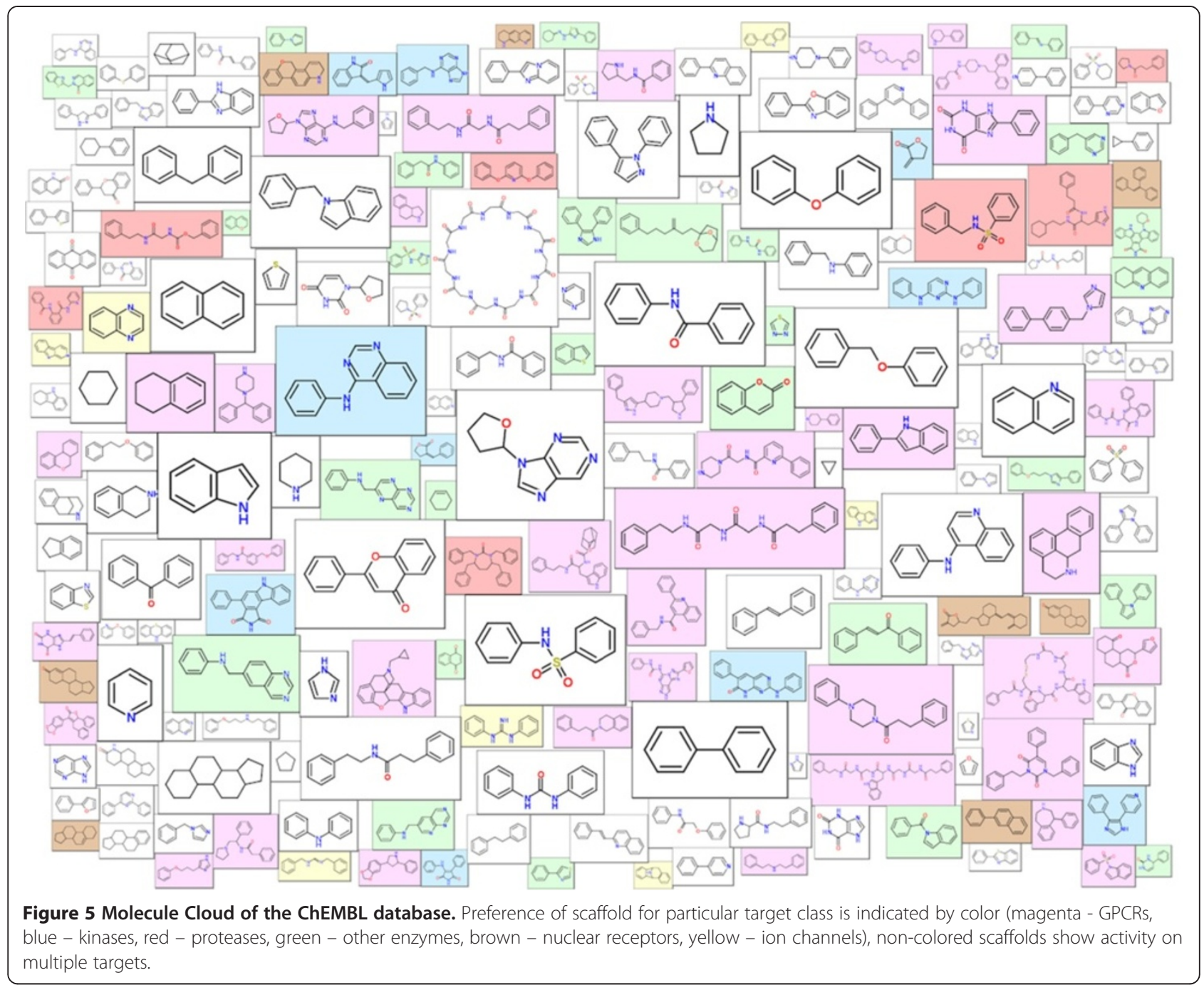

calculated as the sum of overlap areas between molecular frames and the sum of the distances between molecule centers.

In most cases already this initial layout provides quite good placement of molecules. To further improve it, a second layout step is performed, namely an iterative optimization loop. In this step molecules, one by one are slightly moved in the direction that improves the total "overlap score". The convergence is fast and after few seconds the final layout is achieved. During the optimization slight repulsive forces are also placed in the corners of the drawing area to provide aesthetically more pleasing "oval" display instead of completely filling the available image rectangle.

The Molecule Cloud layout algorithm has been implemented in Java. The layout itself does not require any "chemical intelligence", it operates simply on the rectangles representing molecules. The required molecular processing capabilities, particularly parsing of SMILES and molecule depiction are defined by a Java interface class and may be implemented by using any cheminformatics toolkit. The program requires as input only a list of SMILES codes of structures to display with their frequencies and desired size of the final image as input. We tested the algorithm using two cheminformatics engines, the depiction engine from Molinspiration [9] and the recently released Novartis open source Avalon Cheminformatics Toolkit [10]. To interested parties the Java source code of the Molecule Cloud layout algorithm is available from the corresponding author under the terms of the BSD license. The distribution provides also instructions how to interface the program with the Avalon Cheminformatics Toolkit.

\section{Application examples}

In this section Molecule Cloud diagrams are presented for several popular publicly available data sets. PubChem [11] is the largest publicly available molecular structure database. In June 2012 it contained nearly 33 million unique structures. The Molecule Cloud of PubChem is 


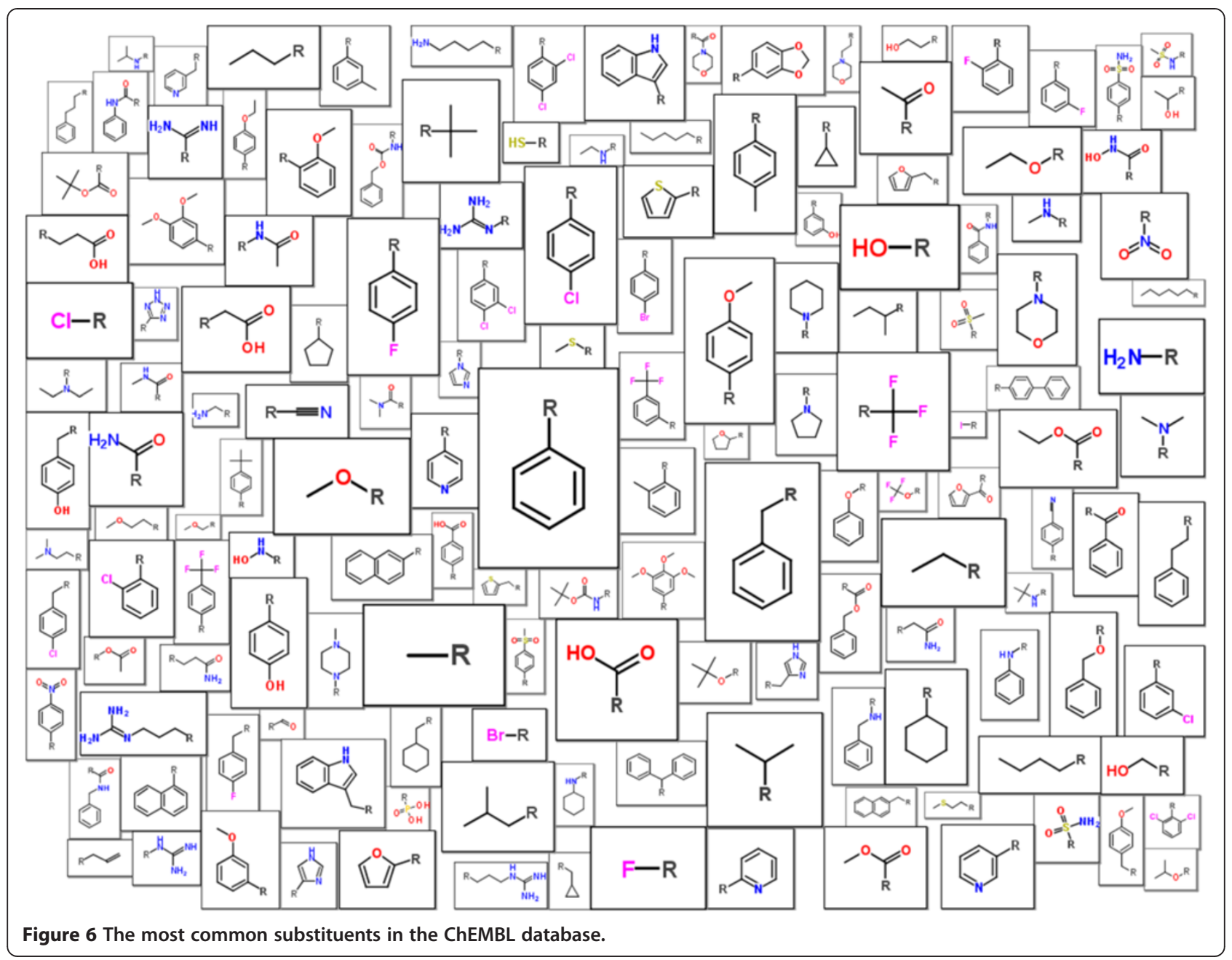

shown in Figure 3. In this image, scaffolds of bioactive molecules are indicated by magenta background, where the color intensity is proportional to the ratio between bioactive and all molecules containing this scaffold. Bioactive molecules were identified by the PubChem advanced search as molecules having activity better than $10 \mu \mathrm{m}$ in any PubChem assay.

A very useful resource for drug discovery, particularly for researchers in academia is the ZINC database [12]. ZINC, created and maintained by John Irwin from the University of California, San Francisco is a collection of commercially available compounds that may be used in virtual screening. The Molecule Cloud graph with the most common scaffolds present in about 12 million ZINC structures is shown in Figure 4.

The last example shows results of analyses of molecules in the ChEMBL database [13]. ChEMBL is a database of molecules extracted from medicinal chemistry journals and other sources. It also contains biological activities and information about the respective targets. This database is an extremely useful source of information particularly for scientists in academia, providing the type of information that was before available only to researchers in pharmaceutical industry. Molecule Cloud visualizing the most common scaffolds of more than 350,000 bioactive molecules (having activity below $10 \mu \mathrm{m}$ ) from ChEMBL is shown in Figure 5. In this figure, information about targets is also displayed by using color. The following six target classes were considered: GPCRs, ion channels, nuclear receptors, kinases, proteases and other enzymes. The scaffold box is colored if at least $70 \%$ of molecules containing this scaffold show activity on particular target class. Scaffolds exhibiting multiple types of activities are not colored. Among these, one can see several known "privileged scaffolds" [14] including biphenyl, indole, quinoline and purine.

The Molecule Cloud allows, of course, visualization of also other structural elements than scaffolds. This is illustrated in Figure 6, where the most common substituents (up to 15 atoms) from the ChEMBL database are shown. Although majority of the substituents are the same as in the other databases [15], the Molecule Cloud 


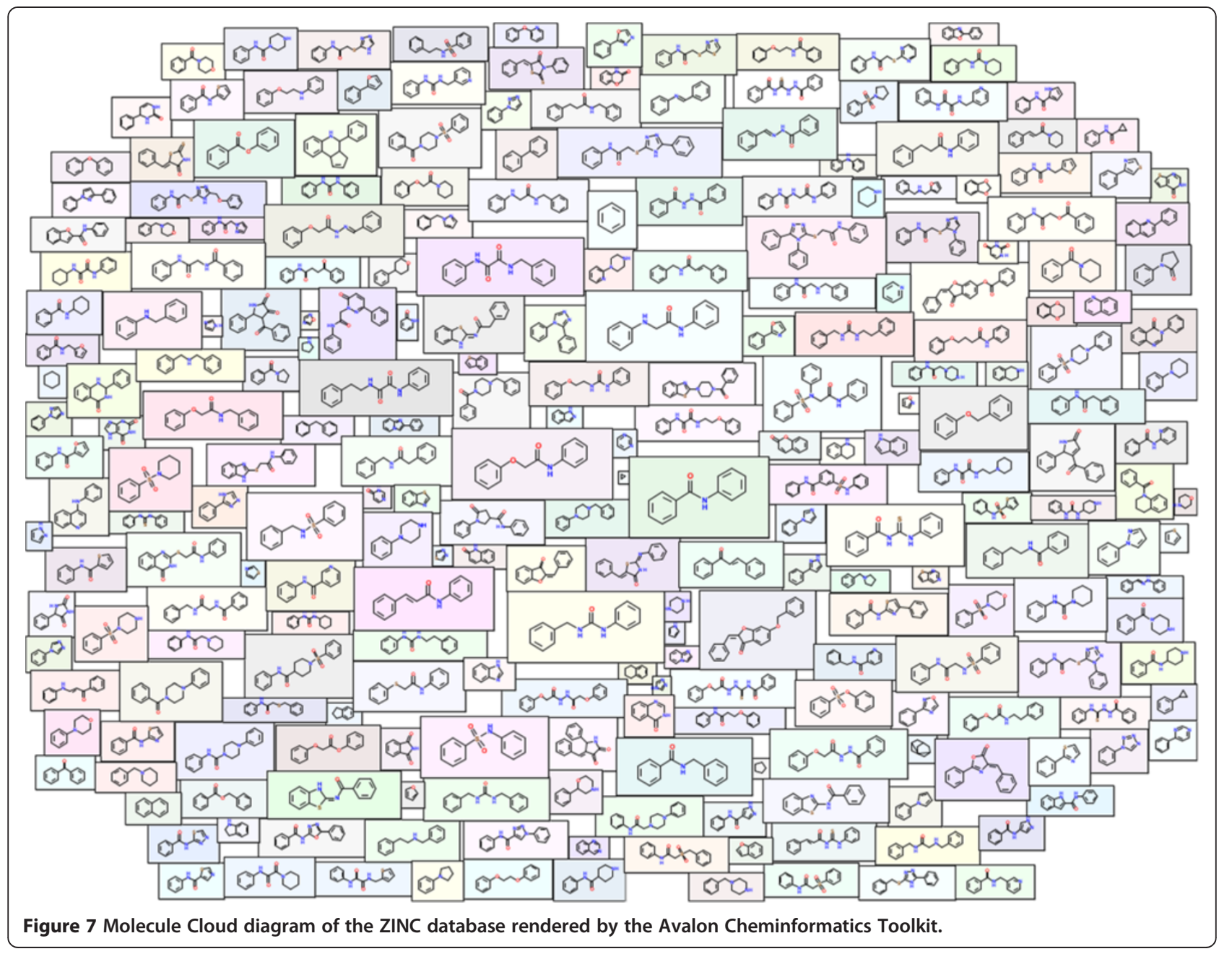

visualization provides clear advantage in comparison with classical molecule grid display.

\section{Conclusions}

Molecule Cloud - a method for compact visualization of the typical substructures present in large collections of molecules was described here. The Molecule Cloud graphs allow to recognize scaffolds and other substructure features that are typical for particular data set by a single look. Additional information about molecules may be included by using colors. Further enhancement of generated images, for example by adding textual information, or possibility to click on particular scaffold to see the molecules containing it, should be with help of HTML5 technology easy.

The Java source code of the Molecule Cloud layout algorithm is available from the corresponding author on request. When combined with the recently released Avalon Cheminformatics Toolkit the software allows generation of Molecule Cloud diagrams (Figure 7) with a completely open source solution.

\section{Competing interests}

The authors declare that they have no competing interests.

\section{Authors' contributions}

PE developed the idea to display set of molecules as a Molecule Cloud in analogy to the visualization of text information as a word cloud and implemented the layout algorithm in Java. BR is author of the Avalon Cheminformatics Toolkit and he interfaced this toolkit with the Molecule Cloud. Both authors read and approved the final manuscript.

Received: 14 May 2012 Accepted: 6 July 2012

Published: 6 July 2012

\section{References}

1. Martin E, Ertl P, Hunt P, Duca J, Lewis R: Gazing into the crystal ball; the future of computer-aided drug design. J Comp-Aided Mol Des 2011, 26:77-79.

2. Langdon SR, Brown N, Blagg J: Scaffold diversity of exemplified medicinal chemistry space. J Chem Inf Model 2011, 26:2174-2185.

3. Blum LC, Reymond J-C: 970 Million druglike small molecules for virtual screening in the chemical universe database GDB-13. J Am Chem Soc 2009, 131:8732-8733.

4. Dubois J, Bourg S, Vrain C, Morin-Allory L: Collections of compounds - how to deal with them? Cur Comp-Aided Drug Des 2008, 4:156-168.

5. Medina-Franco JL, Martinez-Mayorga K, Giulianotti MA, Houghten RA, Pinilla C: Visualization of the chemical space in drug discovery. Cur Comp-Aided Drug Des 2008, 4:322-333. 
6. Schuffenhauer A, Ertl P, Roggo S, Wetzel S, Koch MA, Waldmann H: The Scaffold Tree - visualization of the scaffold universe by hierarchical scaffold classification. J Chem Inf Model 2007, 47:47-58.

7. Langdon S, Ertl P, Brown N: Bioisosteric replacement and scaffold hopping in lead generation and optimization. Mol Inf 2010, 29:366-385.

8. Lipkus AH, Yuan Q, Lucas KA, Funk SA, Bartelt WF, Schenck RJ, Trippe AJ: Structural diversity of organic chemistry. A scaffold analysis of the CAS Registry. J Org Chem 2008, 73:4443-4451.

9. mib 2010.10, Molinspiration Cheminformatics: http://www.molinspiration. com.

10. Bernhard Rohde: Avalon Cheminformatics Toolkit: http://sourceforge.net/ projects/avalontoolkit/.

11. Wang Y, Bolton E, Dracheva S, Karapetyan K, Shoemaker BA, Suzek TO, Wang J, Xiao J, Zhang J, Bryant SH: An overview of the PubChem BioAssay resource. Nucleic Acids Res 2009, 38:D255-D266.

12. Irwin JJ, Shoichet BK: ZINC - a free database of commercially available compounds for virtual screening. J Chem Inf Model 2004, 45:177-182.

13. Gaulton A, Bellis $L$, Bento AP, Chambers J, Davies M, Hersey A, Light $Y$, McGlinchey S, Michalovich D, Al-Lazikani B, Overington JP: ChEMBL: a large-scale bioactivity database for drug discovery. Nucleic Acids Res 2012, 40:D1100-D1107.

14. Welsch ME, Snyder SA, Stockwell BR: Privileged scaffolds for library design and drug discovery. Curr Opin Chem Biol 2010, 14:347-361.

15. Ertl P: Cheminformatics analysis of organic substituents: Identification of the most common substituents, calculation of substituent properties, and automatic identification of drug-like bioisosteric groups. J Chem Inf Comp Sci 2003, 43:374-380.

16. TagCrowd: http://tagcrowd.com.

doi:10.1186/1758-2946-4-12

Cite this article as: Ertl and Rohde: The Molecule Cloud - compact visualization of large collections of molecules. Journal of Cheminformatics 2012 4:12.

\section{Publish with ChemistryCentral and every scientist can read your work free of charge \\ "Open access provides opportunities to our colleagues in other parts of the globe, by allowing anyone to view the content free of charge." \\ W. Jeffery Hurst, The Hershey Company. \\ - available free of charge to the entire scientific community \\ - peer reviewed and published immediately upon acceptance \\ - cited in PubMed and archived on PubMed Central \\ - yours - you keep the copyright \\ Submit your manuscript here: \\ http://www.chemistrycentral.com/manuscript/<smiles>c1ccccc1</smiles> \\ Chemistry Central}

